\title{
El Aprendizaje Significativo Como Estrategia De Estimulación De La Escritura Del Idioma Inglés En Educación General Básica
}

\author{
Daniela Fernanda Guano Merino \\ Magister en Lingüística Aplicada a la Enseñanza Bilingue Español - Inglés \\ Docente de la Escuela Superior Politécnica de Chimborazo, \\ Centro de Idiomas, Ecuador \\ Marcelo Eduardo Allauca Peñafiel \\ Magister en Interconectividad de Redes \\ Técnico Docente de la Escuela Superior Politécnica de Chimborazo, \\ Centro de Idiomas, Ecuador \\ Enrique Jesús Guambo Yerovi \\ Magister en Lingüística Aplicada al Aprendizaje del idioma inglés. \\ Docente de la Escuela Superior Politécnica de Chimborazo, \\ Centro de Idiomas, Ecuador \\ Luis Alberto Veloz Andrade \\ Magister en Administración para el Desarrollo Educativo \\ Docente de la Escuela Superior Politécnica de Chimborazo, \\ Centro de Idiomas, Ecuador
}

doi: 10.19044/esj.2017.v13n19p128 URL:http://dx.doi.org/10.19044/esj.2017.v13n19p128

\begin{abstract}
The objective of the present investigation was the analysis of the use of strategies of significant learning in order to stimulate writing in the English language, which affect the development of the writing skill causing low performance and deficient level of knowledge in the students of sixth and seventh years of general basic education. The bibliographic information on significant learning sustains the importance of the use of didactic material in the processes of assimilation and interaction when acquiring educational skills and competences. They facilitate the connection to preexisting mental schematics in order to incorporate knowledge that generates significant learning. The methods used were deductive-inductive, documentary and applied bibliography, which allowed the collection of information through observation sheets. The results showed that the cognitive processes are inadequate due to lack of didactic material to develop and stimulate the writing of the English language. Due to this worksheets were designed and
\end{abstract}


applied that contain grammatical structure, vocabulary, completion exercises, active memorization, spelling, word classification, sentence formation, short sentences and long with positive and negative answers; use of demonstrative adjectives, possessive pronouns, classification of nouns; all this to stimulate writing and a base of knowledge in the learning of English as a second language.

Keywords: Meaningful learning, stimulating writing strategies, academic contents, educational interaction, cognitive structure, motivation

\section{Resumen}

El objetivo de la presente investigación fue el análisis del uso de estrategias de aprendizaje significativo para la estimulación de la escritura del idioma inglés. La falta de dicha estrategia de aprendizaje afecta al desarrollo de la escritura y que causa bajo rendimiento y deficiente nivel de conocimientos en los estudiantes de sextos y séptimos años de educación general básica. La revisión bibliográfica del aprendizaje significativo, sustentó la importancia de la utilización de material didáctico en los procesos de asimilación e interacción de la adquisición de habilidades y competencias educativas para facilitar la conexión a los esquemas mentales preexistentes, a fin de incorporar conocimientos que generen aprendizaje significativo. Los métodos utilizados fueron el inductivo deductivo, bibliográfico documental y aplicado, que permitió la recopilación de información a través de fichas de observación, los resultados evidenciaron que los procesos cognitivos son inadecuados por falta de material didáctico para desarrollar y estimular la escritura del idioma inglés; por lo que se diseñó y aplicó hojas de trabajo que contienen estructura gramatical, vocabulario, ejercicios de completación, memorización activa, deletreo, clasificación de palabras, formación de oraciones, frases cortas, largas con respuestas positivas, negativas; utilización de adjetivos demostrativos, pronombres posesivos, clasificación de sustantivos; con lo cual se estimuló la escritura y se generó una base de conocimientos en el aprendizaje del idioma inglés como segunda lengua.

Palabras-claves: Aprendizaje significativo, estrategias de estimulación para la escritura, contenidos académicos, interacción educativa, estructura cognitiva, motivación

\section{Introducción}

El aprendizaje significativo como estrategia de estimulación de la escritura del idioma inglés en Educación General Básica tuvo como objetivo, ser un apoyo didáctico para desarrollar habilidades de escritura de la lengua extranjera con el propósito de estimular la adquisición de vocabulario, 
formación de palabras, estructuración de oraciones para que los estudiantes alcancen el perfil de salida de la asignatura de Inglés de acuerdo a los estándares de aprendizaje de la lengua inglesa exigidos por el Ministerio de Educación del Ecuador (2012) para la Educación General Básica. En tal sentido, es necesario estimular a los estudiantes desde los primeros niveles de educación en cuanto al significado, la comprensión, y la producción escrita de la lengua.

Actualmente, aprender el idioma inglés resulta imprescindible puesto que se lo utiliza en casi todas las área del conocimiento y desarrollo humano por lo que puede afirmarse que es la lengua del mundo de la globalización; la lengua internacional en la que el ser humano se comunica en cualquier parte del mundo y que influye directa o indirectamente en todos los campos y profesiones. Por esta razón, el dominio de un buen nivel de inglés, constituye un valor agregado dentro del perfil profesional de una persona.

La implementación del Aprendizaje Significativo como Estrategia de Estimulación de la Escritura del idioma Ingles en Educación General Básica coadyuva al fortalecimiento de la enseñanza de Inglés como lengua extranjera, con el objetivo de alcanzar un nivel funcional de uso de la lengua inglesa para que el estudiante de educación general básica disponga de herramientas comunicacionales universales que le permitan integrarse en cualquier ámbito ya sea profesional, educativo, social y cultural.

Las investigaciones que se han realizado y que apoyan el presente trabajo de investigación, dan a conocer que todos los trabajos están enfocados a la utilización de estrategias para la escritura del inglés a nivel de bachillerato, más no de educación básica; sin embargo, indagando en repositorios digitales de las universidades internacionales, se encontró una investigación dirigida especialmente a la enseñanza del Inglés a nivel primario titulada "El aprendizaje significativo del idioma inglés en Alumnos de Sexto Grado de Primaria a través de Enciclomedia’”, de Jaimes, M. (2012) de la Universidad Pedagógica Nacional de México, Maestría en Pedagogía y Práctica Docente, donde el problema de estudio son las deficientes herramientas básicas para comunicarse en una lengua adicional que utilizan los estudiantes lo que genera dificultades en la secundaria.

\section{Metodología}

El programa de inglés Enciclomedia es un sistema de educación con una base de datos didácticamente diseñada y planeada, a partir de libros de texto para quinto y sexto grados de la educación primaria. A través de una computadora, un pizarrón electrónico y un proyector, los docentes y alumnos tienen acceso en el aula de clases a materiales como: los libros de texto gratuitos, un sitio del docente con el avance programático, objetos de aprendizaje y desarrollo profesional. 
El proceso metodológico se basó en la investigación exploratoria, aplicada y descriptiva bajo un enfoque cualitativo y un diseño de tipo cuasiexperimental que permitió concluir que cada uno de los recursos utilizados en el programa de Inglés de Enciclomedia que permitió estimularon la atención de los niños por aprender de forma divertida y dinámica por la gran variedad de imágenes, frases, ejercicios de ordenación de letras, de búsqueda de letras, palabras, y de selección múltiple que se presenta,

Para el diseño de las estrategias de aprendizaje significativo en la estimulación del desarrollo del Idioma Inglés se utilizó el método inductivo e deductivo. Inductivo debido a que mediante la observación de los hechos particulares se obtuvo las proposiciones generales y deductivas, pues con los resultados generales se llegó a lo particular. Para ello se aplicó las hojas de trabajo a fin de obtener mayor involucramiento del estudiante en el desarrollo de la escritura.

Para el análisis de la información escrita del aprendizaje significativo como estrategia de estimulación de la escritura del idioma inglés. Se utilizó la investigación bibliográfica - documental ya que se pudo conocer, ampliar y profundizar diferentes enfoques, teóricas, conceptualizaciones que fueron aplicados al contexto de la investigación plasmados en el marco teórico. La recopilación de los hechos, datos e información para el avance del conocimiento siguió un proceso estructural específico para resolver el problema del bajo nivel del desarrollo de la escritura en el idioma inglés, además se fundamentó en la investigación aplicada en la información de las fuentes primarias y secundarias.

La descripción de las características importantes del problema de la investigación enmarcada en el bajo nivel de desarrollo de la escritura en el idioma inglés, describe el problema dentro de una circunstancia con espacio temporal determinada; es decir en la Escuela de Educación General Básica Pablo Arturo Suárez, durante el año lectivo 2014-2015 para detallar cómo es y cómo se manifiesta el problema. Se utilizó también la investigación correlacional se verificó el grado de estimulación de la variable independiente aprendizaje significativo en la variable dependiente desarrollo de la escritura del idioma inglés.

\section{Población y muestra}

Se determinó con total exactitud la población con la cual se trabajó, por ello es necesario entender el concepto de población para lograr una mejor comprensión de su significado. Según Diaz (2010), "la población es el conjunto de elementos con características comunes, en un espacio y tiempo determinados, en los que se desea estudiar un hecho o fenómeno.” Bajo este precedente se pudo determinar con exactitud la población para el presente estudio, la cual estuvo conformada de la siguiente manera: 
Tabla 1: Población de sexto y séptimo año de la EGB

\begin{tabular}{ccccccc}
\hline DESCRIPCIÓN & HOMBRES & $\%$ & MUJERES & $\%$ & CANTIDAD & TOTAL \\
\hline Sexto A & 2 & $1 \%$ & 32 & $24 \%$ & 34 & $25 \%$ \\
Sexto B & 1 & $1 \%$ & 33 & $24 \%$ & 34 & $25 \%$ \\
Séptimo A & 1 & $1 \%$ & 34 & $25 \%$ & 35 & $26 \%$ \\
Séptimo B & 1 & $1 \%$ & 31 & $23 \%$ & 32 & $24 \%$ \\
TOTAL & 5 & $4 \%$ & 130 & $96 \%$ & 135 & $100 \%$ \\
\hline
\end{tabular}

Fuente: Directora EGB Pablo Arturo Suárez

Realizado por: Daniela Guano 2017

De acuerdo a los registros de la Dirección de la Escuela Pablo Arturo Suárez, existen 135 estudiantes legalmente matriculados en los sextos y séptimos grados, paralelos A y B durante el año lectivo 2014-2015.

De los estudiantes de los sextos y séptimos años de la Escuela Pablo Arturo Suárez se registraron datos que luego fueron analizarlos e interpretados. Según los medios utilizados, la observación fue estructurada ya que se recurrió a los instrumentos de recopilación de datos diseñados; es decir, se establecieron de los aspectos a estudiar. La participación del observador fue fundamental pues al examinar la población, se logró obtener la información atinente a la investigación sin que sea partícipe directo de la actividad realizada.

Por el tamaño de la población se contó con cinco observadores que trabajaron planificadamente en tiempo y aspecto, en la estimulación del desarrollo de la escritura del idioma inglés, en los paralelos A y B de los sextos y séptimos años de educación básica de la Escuela Pablo Arturo Suárez del Cantón Baños de Agua Santa. Ander-Egg, E. (2009).

La ficha de observación se diseñó para registrar aspectos puntuales de como el aprendizaje significativo estimula la escritura trabajos académicos independientes, actividades individuales y grupales en clase, lecciones y evaluaciones escritas de bloque. En este caso, se utilizó la ficha que consta de 20 criterios relacionados con el problema y variables de estudio, que respondieron a la escala valorativa de Likert (Rensis Likert, 1932), permitió alcanzar los objetivos propuestos, especificando el nivel de acuerdos y desacuerdos de un criterio. Los datos recogidos a través de la observación estructurada, tomando en cuenta los siguientes procedimientos:

- $\quad$ Revisión crítica de la información recogida

- $\quad$ Vaciado de datos

- $\quad$ Tabulación y elaboración de cuadros según variables

- $\quad$ Análisis e interpretación de resultados 


\section{Resultados y discusión}

Mediante los instrumentos de recolección de datos se logró los siguientes resultados aplicados a los estudiantes de Educación General Básica:

La pregunta 1 hace referencia al proceso de clase que fija la forma en que el estudiante aprende en relación al contenido, a sus compañeros y al docente.

Tabla 2: El estudiantado interactúa de forma dinámica en clase.

\begin{tabular}{ccccc}
\hline ESCALA & P01 & Porcentajes & $\begin{array}{c}\text { Porcentajes } \\
\text { acumulados }\end{array}$ & Criterio \\
\hline Siempre & 5 & $10 \%$ & $38 \%$ & Adecuado \\
Generalmente & 14 & $28 \%$ & & \\
Algunas veces & 23 & $46 \%$ & & \\
Rara vez & 7 & $14 \%$ & $62 \%$ & Inadecuado \\
Nunca & 1 & $2 \%$ & & \\
TOTAL $=>$ & 50 & $100 \%$ & $100 \%$ & \\
\hline
\end{tabular}

Fuente: Ficha de Observación

Realizado por: Daniela Guano, 2017

Gráfico 1: Interacción Educativa

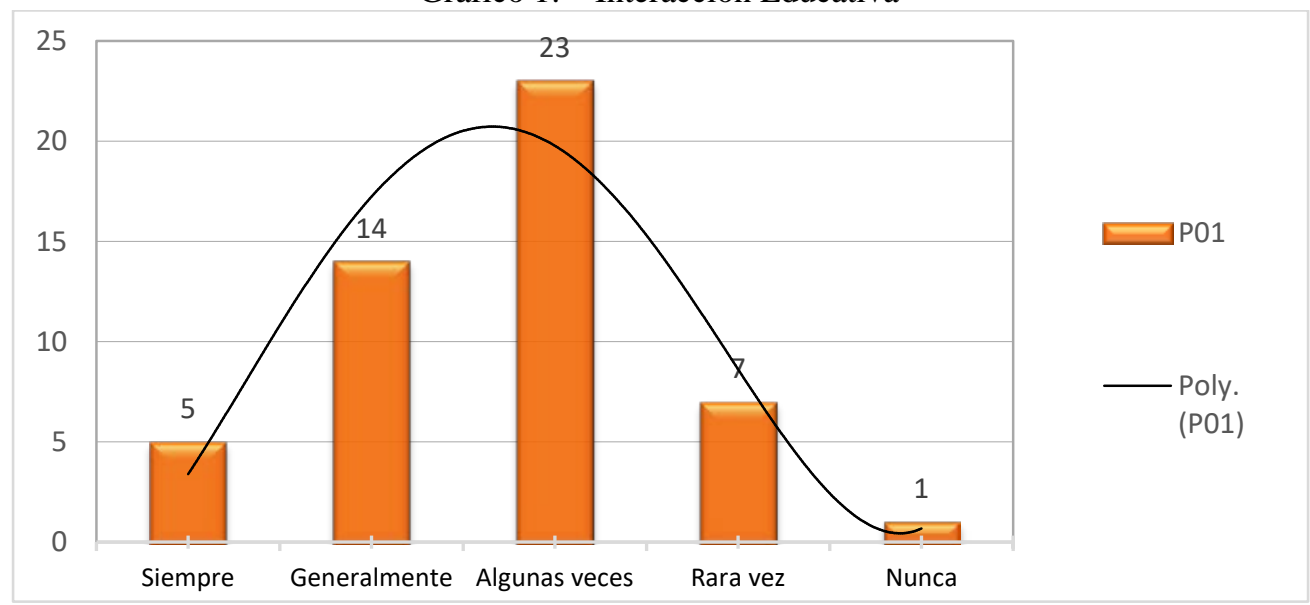

Realizado por: Daniela Guano, 2017

El $62 \%$ de los estudiantes desarrollan un proceso cognitivo inadecuado en la clase inglés, debido a que la interacción entre lo que aprende y el contenido apenas se encuentra en un 38\%; por ello se evidencia también una asimilación e incorporación inadecuada de vocabulario al nuevo conocimiento, lo cual dificulta el avance de la escritura del idioma inglés.

La pregunta 2 hace referencia al proceso cognitivo que posibilita la fijación de la información adquirida y vinculada a conocimientos recientes con los aspectos relevantes y prexistentes. 
Tabla 3: Asimilan fácilmente la escritura del idioma inglés.

\begin{tabular}{ccccc}
\hline ESCALA & P02 & Porcentajes & $\begin{array}{c}\text { Porcentajes } \\
\text { acumulados }\end{array}$ & Criterio \\
\hline Siempre & 3 & $6 \%$ & $24 \%$ & Adecuado \\
Generalmente & 9 & $18 \%$ & & \\
Algunas veces & 31 & $62 \%$ & & \\
Rara vez & 7 & $14 \%$ & $76 \%$ & Inadecuado \\
Nunca & 0 & $0 \%$ & & \\
TOTAL $=>$ & 50 & $100 \%$ & $100 \%$ & \\
\hline
\end{tabular}

Fuente: Ficha de Observación

Realizado por: Daniela Guano, 2017

Gráfico 2: Asimilación en el proceso cognitivo

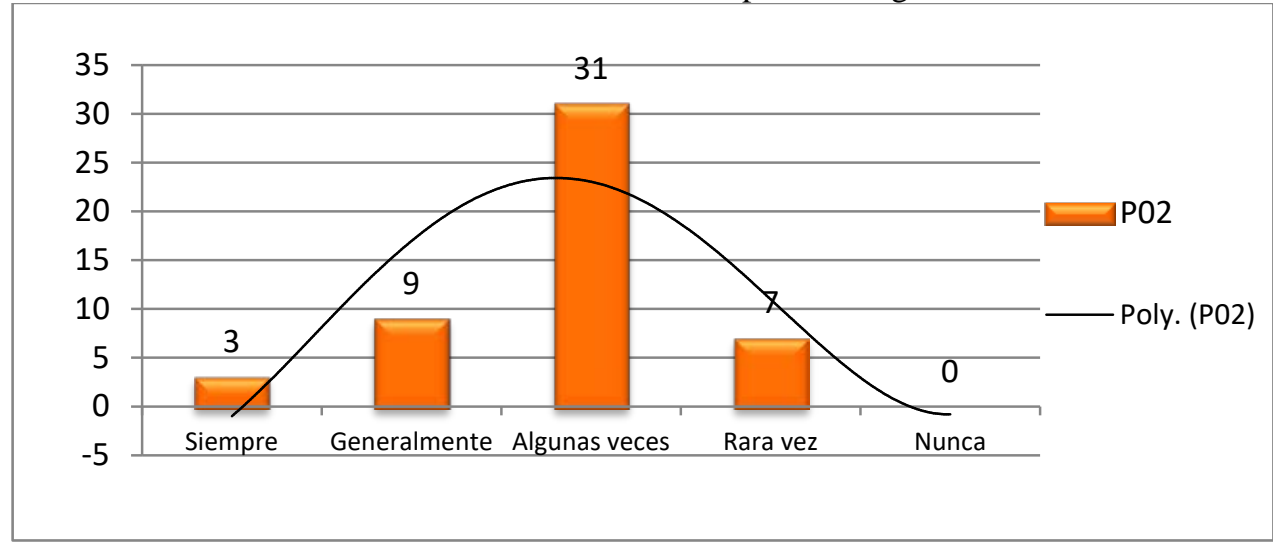

Realizado por: Daniela Guano, 2017

El 76\% de los estudiantes observados asimilan la escritura del idioma inglés de forma inadecuada mientras que $24 \%$ lo hace adecuadamente.

La pregunta 3 hace referencia a la incorporación del proceso cognitivo integrado al conocimiento explícito, que se encuentra en algún tipo de material didáctico con el conocimiento tácito, es decir con lo aprendido gracias a la experiencia.

Tabla 4: Incorporan el nuevo vocabulario escrito al ya existente

\begin{tabular}{ccccc}
\hline ESCALA & P03 & Porcentajes & $\begin{array}{c}\text { Porcentajes } \\
\text { acumulados }\end{array}$ & Criterio \\
\hline Siempre & 3 & $6 \%$ & $22 \%$ & Adecuado \\
Generalmente & 8 & $16 \%$ & & \\
Algunas veces & 12 & $24 \%$ & & \\
Rara vez & 24 & $48 \%$ & $78 \%$ & Inadecuado \\
Nunca & 3 & $6 \%$ & & \\
TOTAL $=>$ & $\mathbf{5 0}$ & $\mathbf{1 0 0 \%}$ & $\mathbf{1 0 0 \%}$ & \\
\hline
\end{tabular}

Fuente: Ficha de Observación

Realizado por: Daniela Guano, 2017 
Gráfico 3: Incorporación de conocimientos

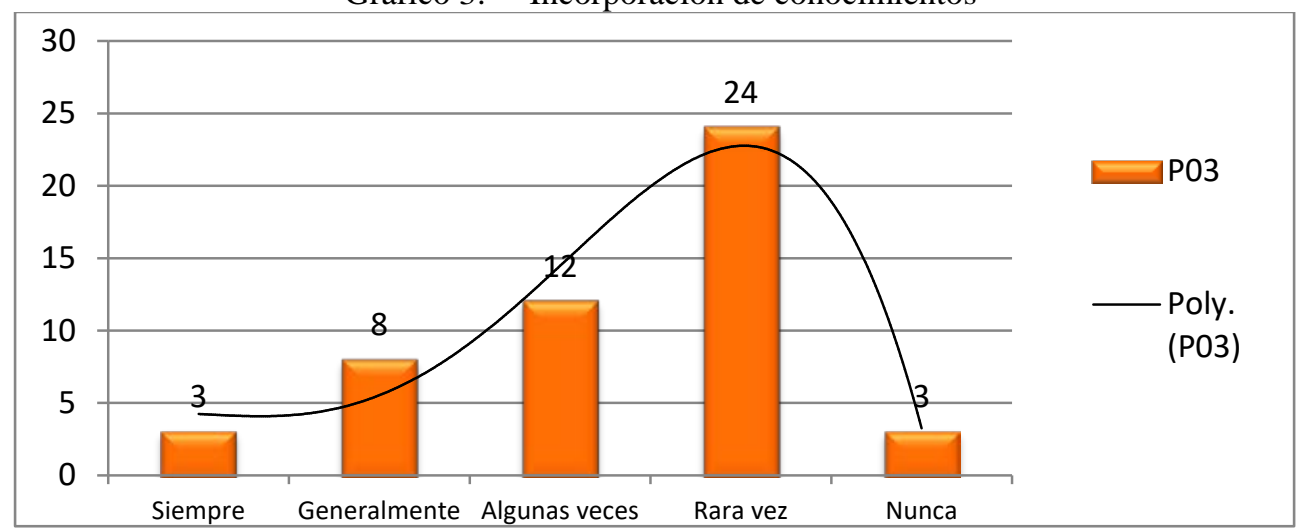

Realizado por: Daniela Guano, 2017

El 78\% de los estudiantes observados incorporan los conocimientos en clase inadecuadamente, el $22 \%$ no lo hace de forma adecuada.

La pregunta 4 hace referencia al proceso lento y requiere más tiempo y estimulación para animar a los estudiantes a que expresen sus ideas en forma escrita

Tabla 1: Captan fácilmente el concepto de una nueva palabra a través de su escritura

\begin{tabular}{ccccc}
\hline ESCALA & P05 & Porcentajes & $\begin{array}{c}\text { Porcentajes } \\
\text { acumulados }\end{array}$ & Criterio \\
\hline Siempre & 3 & $6 \%$ & $32 \%$ & Adecuado \\
Generalmente & 13 & $26 \%$ & & \\
Algunas veces & 27 & $54 \%$ & & \\
Rara vez & 6 & $12 \%$ & $68 \%$ & Inadecuado \\
Nunca & 1 & $2 \%$ & & \\
TOTAL $=>$ & 50 & $100 \%$ & $100 \%$ & \\
\hline
\end{tabular}

Fuente: Ficha de Observación

Realizado por: Daniela Guano, 2017

Gráfico 4: Capta conocimiento nuevo

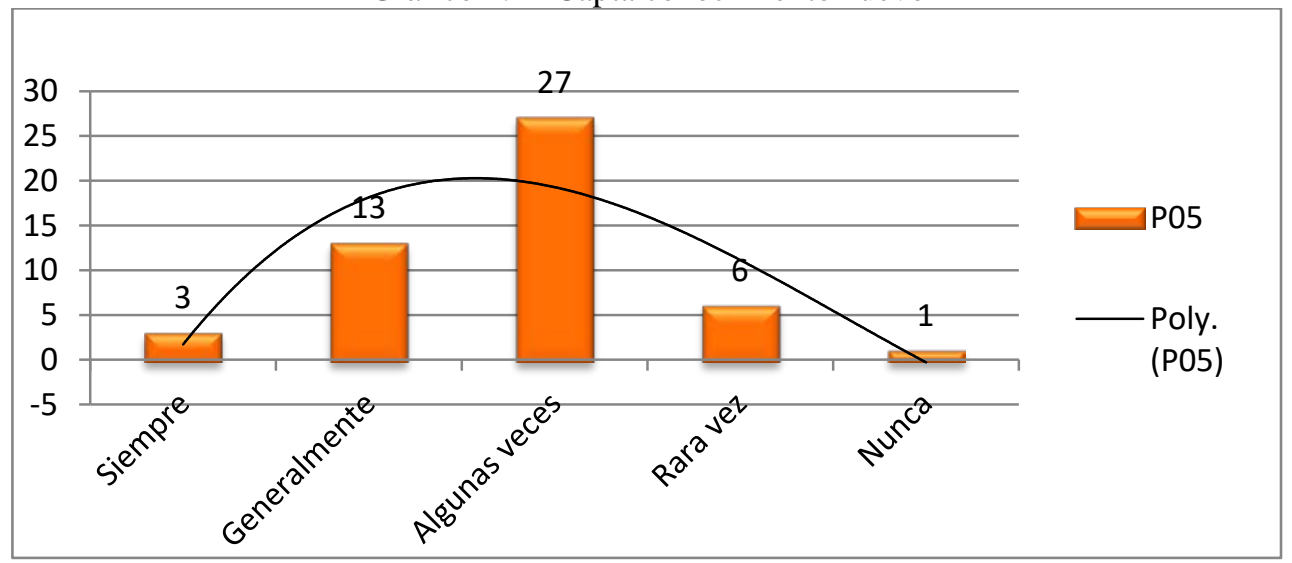

Realizado por: Daniela Guano, 2017 
El 78\% de los estudiantes observados adecuadamente, recuerdan con facilidad la escritura de una nueva palabra, y el 22\% inadecuadamente.

La pregunta 7 hace referencia al concepto o preposición que existe en la estructura cognitiva del estudiante, capaz de servir de anclaje en la adaptación de una nueva información de tal manera que ésta adquiera significado.

Tabla 6: Conectan el nuevo conocimiento con las ideas de anclaje

\begin{tabular}{ccccc}
\hline ESCALA & P07 & Porcentajes & $\begin{array}{c}\text { Porcentajes } \\
\text { acumulados }\end{array}$ & Criterio \\
\hline Siempre & 2 & $4 \%$ & $14 \%$ & Adecuado \\
Generalmente & 5 & $10 \%$ & & \\
Algunas veces & 15 & $30 \%$ & & \\
Rara vez & 24 & $48 \%$ & $86 \%$ & Inadecuado \\
Nunca & 4 & $8 \%$ & & \\
TOTAL $=>$ & 50 & $100 \%$ & $100 \%$ & \\
\hline
\end{tabular}

Fuente: Ficha de Observación

Realizado por: Daniela Guano, 2017

Gráfico 5: Ideas de anclaje.

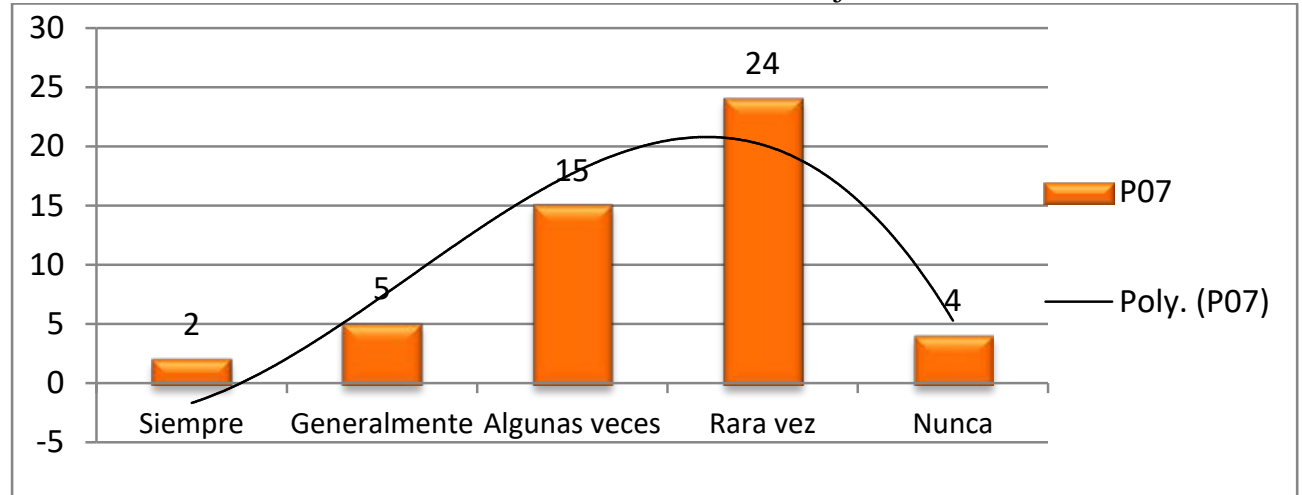

Realizado por: Daniela Guano, 2017

El 86\% de los estudiantes observados conectan inadecuadamente el nuevo conocimiento con las ideas de anclaje en el aula, tan solo el $14 \%$ lo hacen adecuadamente.

La pregunta 9 hace referencia al conjunto de sonidos por medio de los cuales se identifica una palabra, en el idioma inglés y dentro de su aprendizaje es muy importante relacionar la palabra con el sonido, la pronunciación forma parte de las destrezas receptivas y productivas.

Tabla 7: Entienden la pronunciación y escriben fácilmente las nuevas palabras

\begin{tabular}{ccccc}
\hline ESCALA & P09 & Porcentajes & $\begin{array}{c}\text { Porcentajes } \\
\text { acumulados }\end{array}$ & Criterio \\
\hline Siempre & 3 & $6 \%$ & $16 \%$ & Adecuado \\
Generalmente & 5 & $10 \%$ & & \\
Algunas veces & 32 & $64 \%$ & \multirow{2}{*}{$84 \%$} & Inadecuado \\
Rara vez & 9 & $18 \%$ & \\
\hline
\end{tabular}




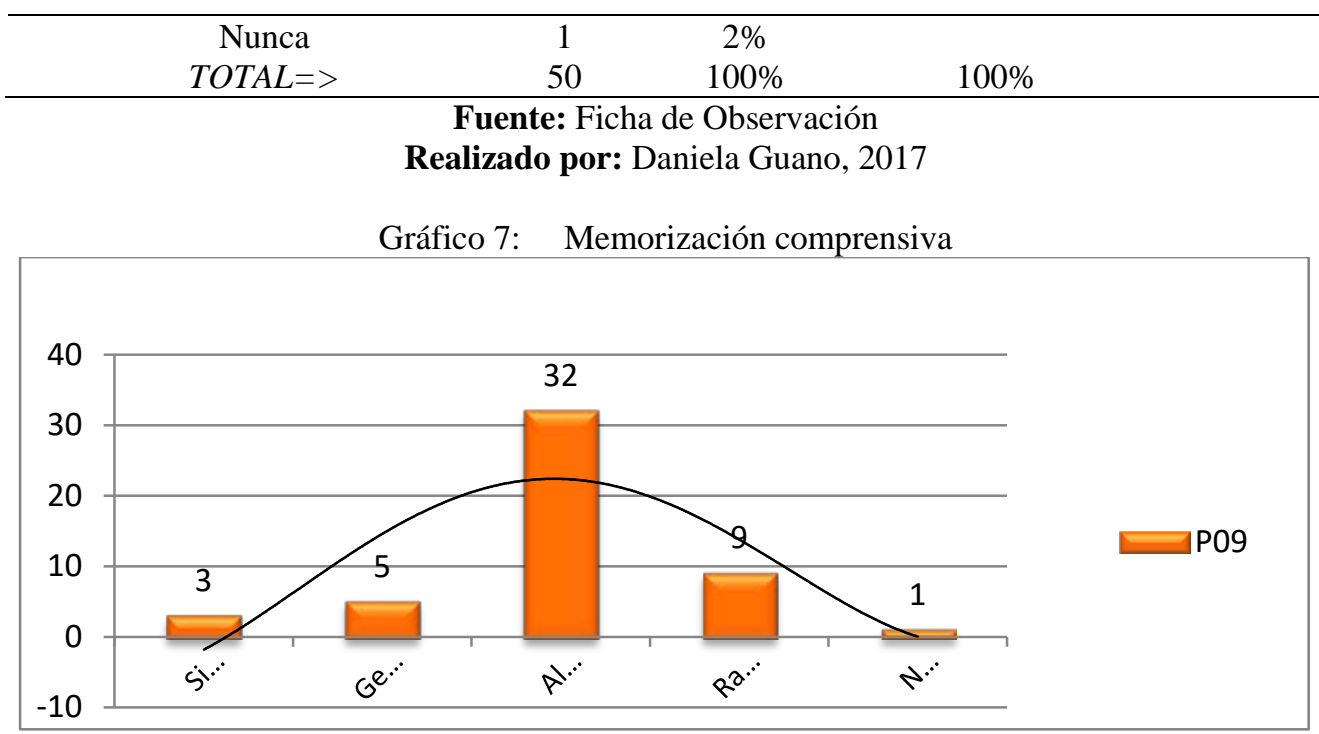

Realizado por: Daniela Guano, 2017

El 84\% de los estudiantes entienden la pronunciación de forma inadecuada mientras que tan solo el 16\% lo hace adecuadamente.

La pregunta 10 hace referencia al interés que tiene el estudiante por su propio aprendizaje o por las actividades que le conducen a él.

Tabla 8: Se motivan con el material que el docente utiliza en el proceso de escritura

\begin{tabular}{ccccc}
\hline ESCALA & P10 & Porcentajes & $\begin{array}{c}\text { Porcentajes } \\
\text { acumulados }\end{array}$ & Criterio \\
\hline Siempre & 3 & $6 \%$ & $20 \%$ & Adecuado \\
Generalmente & 7 & $14 \%$ & & \\
Algunas veces & 32 & $64 \%$ & & \\
Rara vez & 8 & $16 \%$ & $80 \%$ & Inadecuado \\
Nunca & 0 & $0 \%$ & & \\
TOTAL $=>$ & 50 & $100 \%$ & $100 \%$ & \\
\hline
\end{tabular}

Fuente: Ficha de Observación

Realizado por: Daniela Guano, 2017

Gráfico 7: Adquisición de conocimientos

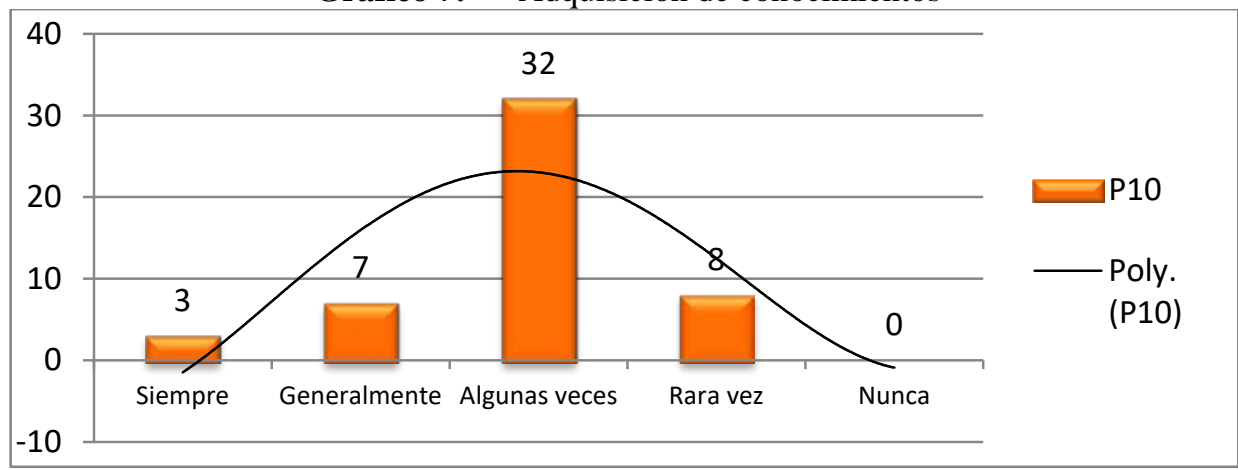

Realizado por: Daniela Guano, 2017 
El $80 \%$ de los estudiantes observados se sienten motivados de forma inadecuada con el material que el docente utiliza mientras que el $20 \%$ lo hacen adecuadamente.

La pregunta 12 hace referencia a los símbolos gráficos que forman el vocabulario y constituye el conjunto de palabras que son parte de un idioma específico.

Tabla 9: Deletrean correctamente una palabra formando frases escritas cortas

\begin{tabular}{ccccc}
\hline ESCALA & P13 & Porcentajes & $\begin{array}{c}\text { Porcentajes } \\
\text { acumulados }\end{array}$ & Criterio \\
\hline Siempre & 4 & $8 \%$ & $22 \%$ & Adecuado \\
Generalmente & 7 & $14 \%$ & & \\
Algunas veces & 14 & $28 \%$ & & \\
Rara vez & 19 & $38 \%$ & $78 \%$ & Inadecuado \\
Nunca & 6 & $12 \%$ & & \\
TOTAL $=>$ & 50 & $100 \%$ & $100 \%$ & \\
\hline
\end{tabular}

Fuente: Ficha de Observación

Realizado por: Daniela Guano, 2017

Gráfico 8: Deletreo de palabras

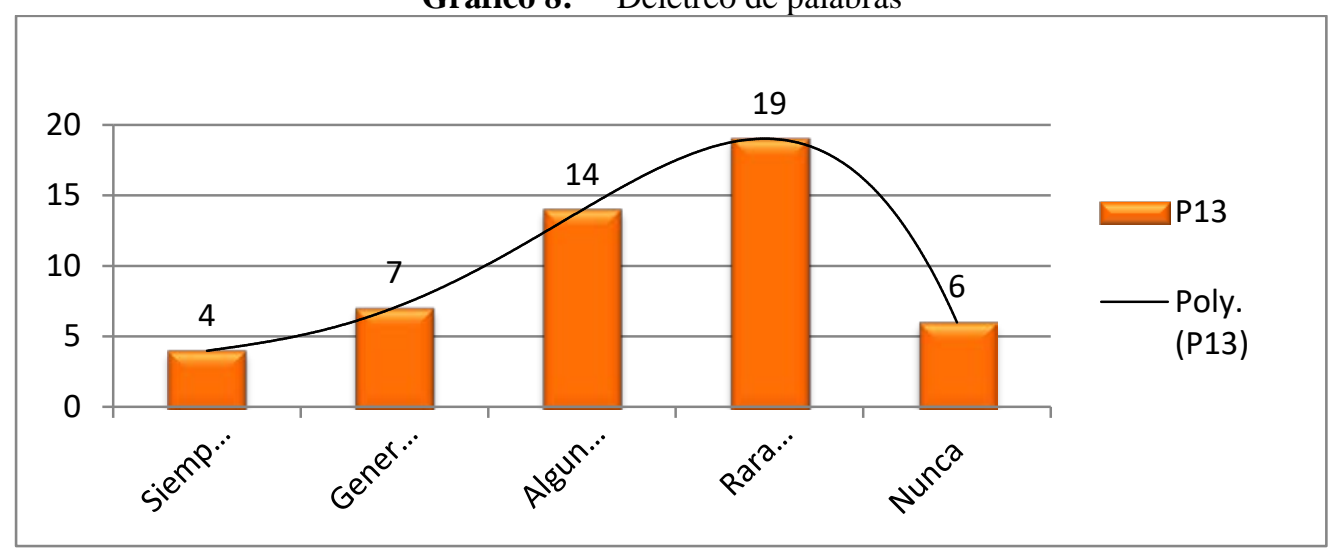

Realizado por: Daniela Guano, 2017

El 88\% de los estudiantes observados utilizan inadecuadamente la ortografía para escribir las palabras en inglés mientras que el $12 \%$ lo hace adecuadamente.

La pregunta 13 hace referencia a la representación gráfica de sonidos, que consiste en una letra o un grupo de letras delimitado por espacios blancos. 
Tabla 10: $\quad$ Se comunican con sus pares a través del proceso de la escritura

\begin{tabular}{ccccc}
\hline ESCALA & P14 & Porcentajes & $\begin{array}{c}\text { Porcentajes } \\
\text { acumulados }\end{array}$ & Criterio \\
\hline Siempre & 1 & $2 \%$ & \multirow{2}{*}{$12 \%$} & Adecuado \\
Generalmente & 5 & $10 \%$ & & \\
Algunas veces & 19 & $38 \%$ & & \\
Rara vez & 18 & $36 \%$ & $88 \%$ & Inadecuado \\
Nunca & 7 & $14 \%$ & & \\
TOTAL $=>$ & 50 & $100 \%$ & $100 \%$ & \\
\hline
\end{tabular}

Fuente: Ficha de Observación

Realizado por: Daniela Guano, 2017

Gráfico 9: Frase o expresión

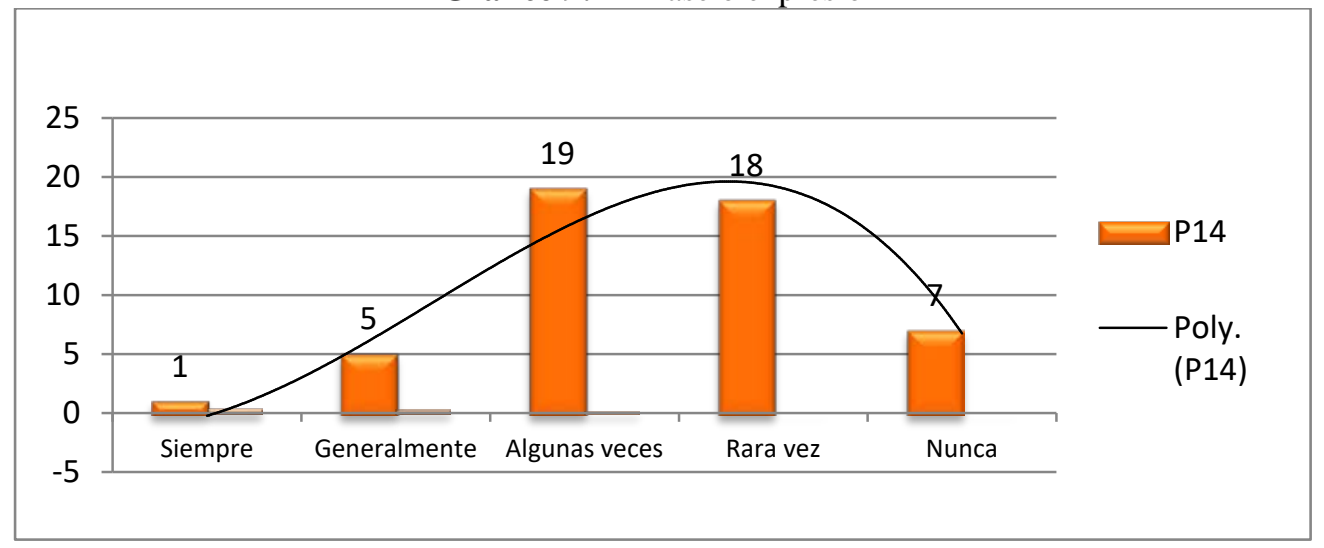

Realizado por: Daniela Guano, 2017

El $78 \%$ de los estudiantes observados adecuadamente deletrean correctamente una palabra formando frases escritas cortas mientras que un preocupante $22 \%$ lo hace de forma inadecuada.

La pregunta 16 hace referencia al interés que tiene el estudiante por su propio aprendizaje o por las actividades que le conducen a él; un factor que siempre debe estar presente durante todo el proceso de estimulación de la escritura en el aula.

Tabla 11: Demuestran curiosidad por completar actividades de completación escrita

\begin{tabular}{ccccc}
\hline ESCALA & P17 & Porcentajes & $\begin{array}{c}\text { Porcentajes } \\
\text { acumulados }\end{array}$ & Criterio \\
\hline Siempre & 3 & $6 \%$ & $26 \%$ & Adecuado \\
Generalmente & 10 & $20 \%$ & & \\
Algunas veces & 19 & $38 \%$ & & \\
Rara vez & 13 & $26 \%$ & $74 \%$ & Inadecuado \\
Nunca & 5 & $10 \%$ & & \\
TOTAL $=>$ & 50 & $100 \%$ & $100 \%$ & \\
\hline
\end{tabular}

Fuente: Ficha de Observación

Realizado por: Daniela Guano, 2017 
Gráfico 10: Curiosidad por la escritura.

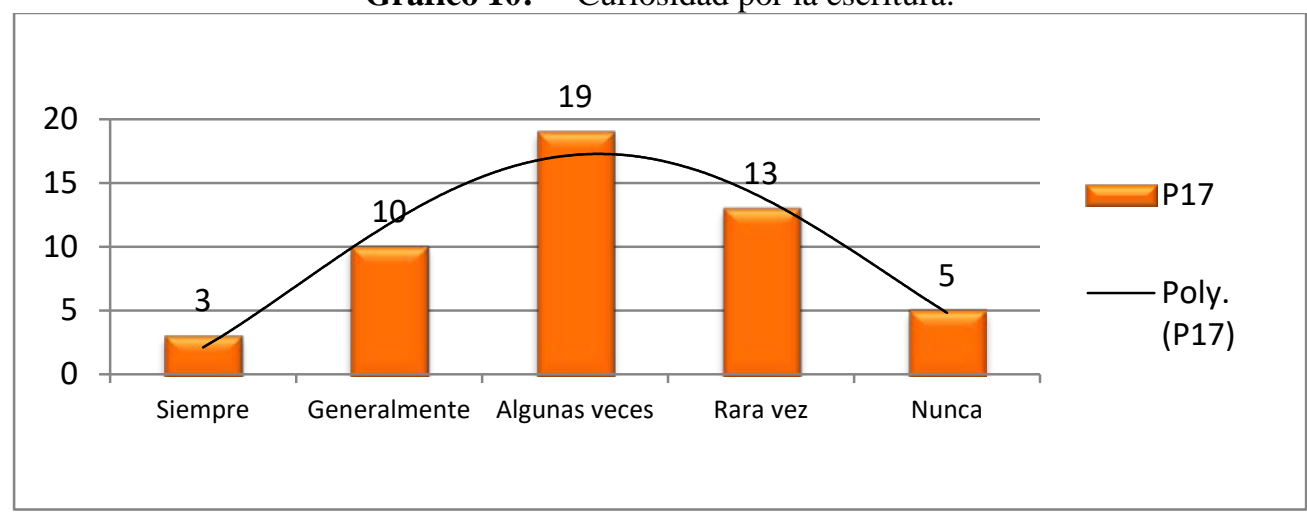

Realizado por: Daniela Guano, 2017

El 68\% de los estudiantes observados demuestran inadecuadamente interés para desarrollar la escritura del inglés, el 32\% lo hace de forma adecuada

\section{Conclusion}

La deficiente escritura se debe a que los estudiantes, no diferencian sonidos para escribir y formar oraciones cortas, razón por la cual no participan activamente en las actividades que propone en el aula.

El desarrollo en el proceso cognitivo inadecuado de los estudiantes en la clase Inglés, se debe a la falta de énfasis entre la interacción lo que aprende y el contenido, evidenciando inadecuado vocabulario al nuevo conocimiento, y a consecuencia de ello dificulta en el avance de la escritura del idioma inglés.

La estructura cognitiva de los estudiantes en el aprendizaje del idioma inglés, carece de conocimiento previo para poder asimilar el nuevo aprendizaje, no permitiendo la adaptación al contexto, con ideas de anclaje basadas en la concepción constructivista y de aprendizaje significativo para incentivar en el estudiante el interés por desarrollar la escritura del idioma inglés en el aula.

En cuanto al contenido escolar que constituye: la funcionalidad, memorización comprensiva, adquisición y retención de conocimientos, existe un nivel inadecuado que evidencia la escasa habilidad del estudiantado para crear estrategias de aprendizaje de interés y desarrollo por la escritura de una lengua extranjera.

La dificultad que tienen los estudiantes en el reconocimiento de los símbolos gráficos, ocasiona problemas como: escaso deletreo, formación de palabras, frases, oraciones cortas. En los niveles educativos elemental y básico, las clases de inglés son cubiertas por los mismos docentes de español, 
lo cual genera más confusión que aprendizaje por la falta de formación profesional y capacitación en el idioma inglés.

El material didáctico es inadecuado y desestimula la curiosidad por la escritura como base de la formación de conceptos y proceso lógicos, escasa comunicación, material didáctico inadecuado, que puede ser motivado con hojas de trabajo que permita el intercambio de ideas que generen formas de participación activa en el proceso de enseñanza aprendizaje de la escritura y del pensamiento que generen de experiencias positivas en el aula.

El programa de Inglés de Enciclomedia estimuló la atención de los niños por aprender de forma divertida y dinámica por la gran variedad de imágenes, frases, ejercicios de ordenación de letras, búsqueda de letras, palabras y de selección múltiple que se presenta. Para ello se aplicó las hojas de trabajo a fin de obtener mayor involucramiento del estudiante en el desarrollo de la escritura mejorando la interacción entre pares.

Los resultados obtenidos demuestran que los sextos y séptimos años de educación general básica de la Escuela Pablo Arturo Suárez interactúan de manera inadecuada en la clase de inglés a pesar de que aplican ideas de anclaje, no logran integrar a su estructura cognitiva el nuevo conocimiento. La deficiente escritura de palabras no permite la formación de oraciones y frases cortas, causando dificultad para deletrear una nueva palabra, incorporar conceptos, lo que genera desinterés hacia el aprendizaje del idioma inglés.

\section{Recomendaciones}

Diseñar hojas de trabajo para el aprendizaje del idioma inglés en Educación General Básica de la Escuela Pablo Arturo Suárez, como recursos de refuerzo y consolidación en una asimilación e incorporación del desarrollo de la escritura del idioma.

Incorporar a las hojas de trabajo con estrategias de aprendizaje para el procesamiento de la información a partir del conocimiento previo que posibilite la acumulación de conceptos que ayuden a la comprensión y memorización de signos de la escritura del idioma inglés.

Integrar a las hojas de trabajo, contenido escolar funcional que aseguren el desarrollo de habilidades de escritura del idioma inglés para el logro de las condiciones enriquecedoras que estimulen el interés por aprender.

Plantear en las hojas de trabajo estrategias de estimulación de reconocimiento de símbolos gráficos, ortográficos para el desarrollo de la escritura del idioma inglés, a través de sopa de letras, crucigramas; ordenación de palabras, de construcción de oraciones, frases; historias, canciones y poemas cortos para que los estudiantes entiendan la 
comunicación escrita e incorporen los signos gráficos y ortografía correcta a su lenguaje diario.

Desarrollar actividades atractivas y motivantes que capten la atención del estudiante y los motiven a aprender el nuevo idioma a través de hojas de trabajo interactivas motivadoras, con gráficos sugerente al tema de clase que suscite e incremente el interés por aprender la escritura, la pronunciación, la formación de palabras, frases y oraciones del idioma inglés.

Propiciar a través de las hojas de trabajo actividades que generen interacción educativa entre los estudiantes para el incremento de la comunicación en la segunda lengua extranjera estimulando el desarrollo de la escritura efectiva en la lengua extranjera.

\section{References:}

1. Alvarado, R. (2013). El aprendizaje Significativo.Importancia de los conocimientos propios de los estudiantes. Obtenido de https://maestrociro.wordpress.com/2010/08/22/el-aprendizajesignificativo-importancia-de-los-conocimientos-previos-de-losestudiantes/

2. Alvarez, R. (2011). Desarrollo de los procesos (aprendizajememorización).

Obtenido

de

http://www.monografias.com/trabajos91/aprendizaje-procesoscognitivos-observacion-comparacion-resumen/aprendizaje-procesoscognitivos-observacion-comparacion-resumen.shtml\#procesosca

3. Ander-Egg, Ezequiel. (2009). Técnicas de Investigación Social. Obtenido de https://imas2009.files.wordpress.com/2009/04/anderegg_135-175.pdf

4. Ariagno, D. (2012). Evolución de la escritura y de sus soportes. Obtenido de http://www.grafotrends.com/uploads/2/6/6/3/26632599/evolucion_de _la_escritura_y_sus_soportes.pdf

5. Azzi, Milagros. (2013). Desarrollo de los procesos cognitivos. Obtenido http://milagrosazzi.aprenderapensar.net/author/milagrosazzi/

6. Campistrous, L. (2009). Lógica y procedimientos lógicos del pensamiento. fdeportes, 15.

7. Concha, V. (2014). Ausubel y el aprendizaje significativo. Obtenido de

http://www.rmm.cl/index_sub.php?id_seccion=1122\&id_portal=191 \&id_contenido=988

8. Cook, T.D. (1986). Métodos cualitativos y cuantitativos en la investigación evaluativa. Madrid: Morata, S. L.

9. Díaz, F. (2010). Técnicas de Elaboración de Tesis. Ambato: UTA. 
10. Díaz, F. (2012). Estrategias Docentes para un aprendizaje Significativo. Una interpretación constructivista. México: McGraw Hill.

11. Díaz, F., \& Rojas, G. (2010). El aprendizaje de diversos contenidos curriculares. McGraw Hill.

12. Echeita Gerardo \& Minguillón Carmen. (2010). "Las necesidades ducativas especiales del niño con deficiencia auditiva". Ministerio de Educación y Ciencia.

13. Education First. (28 de Enero de 2014). Ecuador está entre los países con nivel más bajo de Inglés. El Telégrafo.

14. Fingermann, Hilda. (2010). Los contenidos escolares. La guía.

15. Gamonal, Carlos. (2011). Metodología del trabajo intelectual. Obtenido de http://serestudianteeficaz.blogspot.com/2011/06/desarrollo-de-lamemoria-comprensiva.html

16. Gómez, Marcelo. (2006). Introducción a la Metodología de la Investigación Científica. Córdova: Brujas.

17. Gonzales, F. (1979). El Mapa Conceptual y el Diagrama V.

18. Herrera, L. (2008). Tutoría de la Investigación Científica. Ambato: Empresdane Gráficas Cía. Ltda.

19. Herrera, R. (2008). La Teoría del aprendizaje de Vygotski. Revista de Acción pedagógica y curricular. Innovar en educación.

20. Hersh, R., Paolitto, D., \& Reimer, J. (2012). El crecimiento moral. Madrid: Narcea S. A.

21. Jackson, Anne. (2008). Como estimular a escribir a los niños. En C. d. WestEd, Niños de la edad preescolar que aprenden ingles (pág. 86). Clifornia: Guia de Recursos. Departamiento de Eduacacion de Clifornia.

22. Kupka, Tomás. (2013). Reflexiones sobre la inexistencia de la base teórica de la creación y evaluación de las hojas de trabajo para la enseñanza de lenguas extranjeras con un enfoque en la lengua española. Dialnet, 121-125.

23. Lamanier, H. (2014). Gestión del conocimiento, definiciones y características del conocimiento. La Habana-Cuba.

24. Lorenzo, F. (2006). Motivación y Segundas Lenguas. Madrid: Arco Libros.

25. Lozano, W. (Noviembre de 2008). La estructura cognitiva sobre la base de la Teoría de Ausubel. Obtenido de http://williams.blogspot.es/categoria/general/

26. Manosalvas, J. (2010). Las técnicas activas utlizadas para desarrollar la destreza de la escritura del Inglés. Obtenido de 
http://repositorio.ute.edu.ec/bitstream/123456789/12074/1/41832_1.p df

27. Manosalvas, José. (2010). Técnicas activas utilizadas para desarrollar la destreza de la escritura del Inglés. Obtenido de http://repositorio.ute.edu.ec/bitstream/123456789/12074/1/41832_1.p df

28. Marín Ibáñez, R. (2004). El aprendizaje abierto y a distancia, el material impreso. Loja: RIED.

29. Marín, L. (2012). Naturaleza de la investigación. Obtenido de http://www.sc.ehu.es/plwlumuj/kualitatiboa/powerpointcualitativo/1\%20Naturaleza\%20de\%20la\%20investigacion.pdf

30. Marín, N. (2007). Fundamentos . Almería: Universidad Almería.

31. Mejía, Pablo. (2013). Contrastive phonology essentials. Quito: Systemgrafic.

32. Nuñez, José de Jesús. (2003). Interacción grupal y psicopatología. México: Plaza y Valdez.

33. Ortiz, A. (2005). Violencia doméstica: Modelo multidimensional y programa de intervención. Obtenido de http://biblioteca.ucm.es/tesis/fsl/ucm-t28681.pdf

34. Panez, J. (Diciembre de 2014). ¿Cómo estimular la escritura en la etapa preescolar. Obtenido de http://app.kiddyshouse.com/maestra/articulos/como-estimular-laescritura-en-la-etapa-preescolar.php

35. Piaget, J. (2001). La formación de la inteligencia. México.

36. Posso, Miguel. (2011). Proyectos, Tesis y Marco Lógico. Planes e informes de investigación. Quito: Noción Imprenta.

37. Ramírez, Jesús. (2010). Conocimientos previos. Proyecto educativo, 3.

38. Rodríguez, Ma. Luz. (2004). La Teoría del Aprendizaje Significativo. Obtenido de http://cmc.ihmc.us/papers/cmc2004-290.pdf.

39. Samaniego, A. (2013). Las teorías del aprendizaje significativo que aplican los docentes de Ciencias Naturales en la Planificación Micro Curricular y su incidencia en el Desarrollo de destrezas. Obtenido de http://dspace.unl.edu.ec/jspui/bitstream/123456789/2792/1/SAMANI EGO\%20QUEZADA\%20ANA\%20LUCIA.pdf

40. Santamaría, S. (2015). Teorias de Piaget. Obtenido de http://www.monografias.com/trabajos16/teorias-piaget/teoriaspiaget.shtml

41. Tulchán, Edwin. (2015). Diseño de una estrategia didáctica de aprendizaje significativo para mejorar la lectura de inglés para propósitos específicos en una Institución Aeronáutica. Obtenido de http://repositorio.pucesa.edu.ec/jspui/handle/123456789/1152 
42. Vigotsky, L. (1987). La Historia de las funciones psíquicas superiores. La Habana: Científico-Técnica.

43. Viu.es. (2015). Universidad Internacional de Valencia. Obtenido de http://www.viu.es/el-aprendizaje-funcional-en-el-aula-ordinaria/

44. Yugla, Rebeca. (2015). Diseño de Recursos Didácticos visuales para el desarrollo de habilidades comunicativas del Idioma inglés de los estudiantes de bachillerato. Obtenido de repositorio.pucesa.edu.ec/jspui/bitstream/123456789/1474/1/75965.p df

45. Zeitler, T. (2013). Historia de la escritura y el diseño I, desde la antiguedad hasta lal modernidad. Madrid: Akal. 